\title{
Inhibition of the PI3K/Akt pathway increases the chemosensitivity of gastric cancer to vincristine
}

\author{
$\mathrm{XIA} \mathrm{XIE}^{1^{*}}$, BO TANG $^{1 *}, \mathrm{JIANYUN} \mathrm{ZHOU}^{1}$, QING GAO $^{2 * *}$ and PENGBING ZHANG ${ }^{\text {*** }}$ \\ ${ }^{1}$ Department of Gastroenterology, Xinqiao Hospital, Third Military Medical University, Chongqing 400037; ${ }^{2}$ Department of \\ Gastroenterology, First Affiliated Hospital, Chongqing Medical University, Chongqing 400016, P.R. China
}

Received March 13, 2013; Accepted May 8, 2013

DOI: $10.3892 /$ or.2013.2520

\begin{abstract}
The phosphatidylinositol 3-kinase (PI3K)/Akt signaling pathway plays a crucial role in tumorigenesis and tumor progression by promoting cell proliferation and inhibiting apoptosis, a process closely associated with multidrug resistance (MDR) of tumors. LY294002 is a commonly used pharmacological inhibitor that acts at the ATP-binding site of the PI3K enzyme, selectively inhibiting the PI3K/Akt pathway. In the present study, we evaluated the effect of LY294002 on the chemosensitivity of gastric cancer cells to vincristine (VCR) in vitro and in vivo and investigated the possible underlying cellular mechanisms. The effect of LY294002 on cell viability, apoptosis induction and inhibition of tumor growth was analyzed using MTT and TUNEL assay in in vitro and in vivo models of gastric cancer. Intracellular accumulation of VCR was determined by high performance liquid chromatography. The activity of the PI3K/Akt pathway was evaluated by western blot analysis. Furthermore, reverse transcription PCR and immunohistochemistry were performed to determine the mRNA and protein expression levels of $M D R 1 / \mathrm{P}$-glycoprotein (P-gp) and apoptosis-related factors. We found that gastric cancer cells treated with LY294002 showed a significant inhibition of PI3K/Akt activity. The PI3K inhibitor LY294002 combined with VCR worked synergistically to promote growth inhibition, induce apoptosis and increase the intracellular drug accumulation in gastric cancer cell lines. Similarly, LY294002 could cooperate with VCR to reduce tumor growth
\end{abstract}

Correspondence to: Professor Pengbing Zhang, Department of Gastroenterology, Xinqiao Hospital, Third Military Medical University, 183 Xinqiao Street, Chongqing 400037, P.R. China E-mail: zpb666@sina.com

Professor Qing Gao, Department of Gastroenterology, First Affiliated Hospital, Chongqing Medical University, 1 Youyi Road, Chongqing 400016, P.R. China

E-mail: gaoqing27@yahoo.com.cn

*Contributed equally as first authors

${ }^{* *}$ Contributed equally as senior authors

Key words: multidrug resistance, gastric cancer, phosphatidylinositol 3-kinase/Akt, LY294002, vincristine in a gastric cancer model in vivo. Finally, LY294002 was able to decrease the expression of $M D R 1 / \mathrm{P}-\mathrm{gp}, \mathrm{Bcl}-2$ and XIAP, and upregulate expression of Bax and caspase-3, thereby enhancing chemosensitivity to VCR by inhibiting a drug pump and inducing apoptosis. These results suggested that the PI3K/Akt inhibitor LY294002 can enhance chemosensitivity of human gastric cancer to VCR. This preclinical evaluation of a rational combination of LY294002 and VCR may provide a new strategy to resolve the MDR of gastric cancer.

\section{Introduction}

Gastric cancer is the second most common cancer worldwide and the fourth leading cause of cancer-related mortality $(1,2)$. The incidence, diagnostic studies and therapeutic options have undergone major changes in recent years, but the prognosis for gastric cancer remains poor, particularly in more advanced stages (3). Currently, the delivery of chemotherapeutic agents following surgical resection defines the standard treatment for this malignancy. However, one clinically significant problem that often results in the failure of chemotherapy is multidrug resistance (MDR), which severely limits the effectiveness of chemotherapy in gastric cancer and is responsible for the overall poor efficacy of cancer therapy (4).

MDR is generally used to describe a resistance phenotype where resistance, either inherent or acquired, develops not only to a single cytotoxic agent but also to a whole range of drugs with different structures and cellular targets $(4,5)$. The mechanisms of drug resistance that can act individually or synergistically are complicated, and have been described and extensively studied over the past decades. These include reduction of intracellular drugs by increasing drug efflux and/or decreasing drug uptake $(6,7)$, increased repair of drug-induced DNA damage $(8,9)$, evasion of drug-induced apoptosis $(10,11)$, disruptions in signaling pathways and some other changes in the factors that regulate the resistance mechanisms. Chemotherapeutic drugs induce a series of cellular responses that have an impact on tumor cell proliferation and survival. Furthermore, several studies have suggested a direct correlation between alterations in survival pathways and chemoresistance, and certain components of these pathways have been identified as critical targets for cancer intervention (12). Of these survival pathways, the phosphatidylinositol 3-kinase (PI3K)/Akt signaling pathway plays a major role not 
only in tumor development but also in the potential response of the tumor to treatment.

The PI3K/Akt signal transduction pathway plays a crucial role in tumorigenesis and tumor progression by promoting cell proliferation and inhibiting apoptosis. The PI3Ks are lipid kinases, which can be activated downstream of receptor tyrosine kinases, including epidermal growth factor receptor (EGFR), vascular endothelial growth factor receptor (VEGFR) and insulin-like growth factor receptor (IGFR) $(13,14)$. Following PI3K activation, phosphoinositol-4,5-bisphosphate (PIP2) is converted into the second messenger phosphoinositol-3,4,5-trisphosphate (PIP3) on the inner side of the plasma membrane (15). Then, the lipid product of PI3K and PIP3 recruits a subset of signaling proteins with pleckstrin homology $(\mathrm{PH})$ domains to the membrane, including PDK1 and Akt/PKB (15). Once activated, Akt mediates the activation and inhibition of several targets, resulting in cellular survival, growth and proliferation through various mechanisms. Thus, inhibition of PI3K or molecules involved in the PI3K signaling pathway is a promising approach to treat tumors. Moreover, several studies have indicated that LY294002, a commonly used pharmacological inhibitor of PI3K, could decrease tumor growth, inhibit tumor invasion and migration, and sensitize various tumors to chemotherapy (16-19). Since PI3K/Akt is found to be highly activated in gastric cancer and is positively correlated to progression and chemoresistance of gastric cancer, we hypothesized that treatment with LY294002 may inhibit proliferation and increase the sensitivity of gastric cancer to chemotherapy.

In the present study, we evaluated the effect of inhibition of the PI3K/Akt pathway on the chemosensitivity of gastric cancer cells in vitro and in vivo and investigated the possible underlying cellular mechanisms. Here, we present evidence that LY294002 can enhance chemosensitivity of human gastric cancer cells to vincristine (VCR) by inactivation of the PI3K/Akt signaling pathway. Our findings suggest that modulation of the PI3K/Akt pathway may present a new strategy to improve current therapeutic regimens and provide a molecular basis for the novel design of combination treatments for gastric cancer.

\section{Materials and methods}

Ethics statement. The experiments involving the use of laboratory animals were carried out in accordance with the Animal Welfare Act and the recommendations of the Institutional Animal Care and Use Committee of the Third Military Medical University. All animal studies were approved by the Ethics Committee of Xinqiao Hospital of the Third Military Medical University (Chongqing, China). The animals were humanely sacrificed and all efforts were made to ameliorate suffering.

Cell lines and animals. A moderately differentiated stomach adenocarcinoma cell line, SGC-7901, was purchased from the cell bank of the Chinese Academy of Sciences (Shanghai, China). SGC-7901/VCR, a VCR-resistant cell line, was established and generously gifted by Dr Daimin Fan (Institute of Gastroenterology, Xijing Hospital, The Fourth Military Medical University, China). The SGC-7901 cells were main- tained in RPMI-1640 medium supplemented with $10 \%$ fetal bovine serum (both from Gibco-BRL, Carlsbad, CA, USA), SGC-7901/VCR cells were cultured in the same medium with an additional $1 \mathrm{mg} / \mathrm{VCR}$. All cells were cultured at $37^{\circ} \mathrm{C}$ in a humidified atmosphere containing $5 \% \mathrm{CO}_{2}$.

Twenty-four nude male mice (four weeks old) were purchased from the Shanghai Experimental Animal Center (Shanghai, China) and were maintained in a specific pathogenfree environment, fed with a regular diet.

Western blot analysis. A western blot analysis was used to confirm the inhibition of PI3K/Akt activity in SGC-7901 and SGC-7901/VCR cells. SGC-7901 and SGC-7901/VCR cells were collected by centrifugation and washed twice with PBS. Proteins from these cell extracts were denatured by boiling for $10 \mathrm{~min}$. Protein concentration was determined using the bicinchoninic acid protein assay (Pierce Biochemicals, Rockford, IL, USA) and equal amounts of protein were loaded on a $10 \%$ SDS-PAGE gel and transferred onto a nitrocellulose membrane (Millipore, Bedford, MA, USA) by electroblotting. Subsequently, the membranes were blocked with 5\% non-fat milk in PBS, and incubated with primary antibodies for anti-Akt (Upstate, Billerica, MA, USA), antiphospho-Akt (Cell Signaling Technology, Danvers, MA, USA) or anti- $\beta$-actin mAb (Sigma, St. Louis, MO, USA) at $4^{\circ} \mathrm{C}$ overnight. The membranes were washed and incubated with an alkaline phosphatase-conjugated goat anti-mouse IgG antibody (Amersham Biosciences, Buckinghamshire, UK) for $1 \mathrm{~h}$ at room temperature. Immunoreactive bands were detected using the ECL western blot analysis system (Amersham Biosciences).

Cell viability assay. Cell proliferation was determined by the MTT assay as previously described (19). Briefly, SGC-7901 and SGC-7901/VCR cells were divided into three groups: VCR, LY294002, and VCR + LY294002, respectively. Cells were digested and plated in 96-well plates at a density of $5 \times 10^{3}$ cells/well. The cells in different groups were then treated with varying concentrations of VCR and/or LY294002. Untreated cells were considered as negative control. After $72 \mathrm{~h}$ of incubation, the medium was removed and replaced with $20 \mu \mathrm{l} \mathrm{MTT}(5 \mathrm{mg} / \mathrm{ml})$, and incubated for $4 \mathrm{~h}$ at $37^{\circ} \mathrm{C}$. Then, the supernatant was carefully removed and DMSO was added to each well to dissolve the crystals by gentle agitation for $10 \mathrm{~min}$. The absorbance at $570 \mathrm{~nm}\left(\mathrm{~A}_{570}\right)$ of each well was read on a Microplate Reader (Bio-Tek ELX-800; BioTek, Winooski, VT, USA). The survival rate of cells in each well was calculated according to the following formula: survival rate $=\left(\mathrm{A}_{570}\right.$ of treated wells $/ \mathrm{A}_{570}$ of untreated wells) x $100 \%$. Finally, $\mathrm{IC}_{50}$ values were determined. Each experiment was performed in quadruplicates.

High performance liquid chromatography (HPLC). The cells were plated at a density of $2 \times 10^{7}$ cells $/ 10 \mathrm{~cm}$ dish. On the following day, the cells were treated with VCR combined with LY294002 or not for 3 and 6 h, respectively. After the incubation, the cells were washed three times with cold PBS, collected by digestion and centrifugation, and then resuspended in saline in $2 \mathrm{ml}$ Eppendorf tubes. Cells were broken down by repeated freezing and thawing and then centrifuged 
for $15 \mathrm{~min}$ at $16,000 \mathrm{rpm}$. Subsequently, $20 \%$ perchloric acid (50 $\mu 1 / 500 \mu 1$ of PBS) was added to denature the protein and $1 \mathrm{ml}$ of diethyl ether was added to each tube and mixed by vortexing for $1 \mathrm{~min}$. Following centrifugation for $15 \mathrm{~min}$ at $16,000 \mathrm{rpm}$, the organic phase $(500 \mu \mathrm{l})$ was collected and evaporated to dryness. The dried residue was reconstituted with $500 \mu \mathrm{l}$ of the mobile phase (93\% methanol, $7 \%$ water and $0.18 \%$ triethylamine) and used for HPLC. Samples were analyzed using a C-18 reverse phase column and eluted isocratically with a mobile phase of water and triethylamine in methanol. The flow rate was set at $0.5 \mathrm{ml} / \mathrm{min}$ and column temperature was $40^{\circ} \mathrm{C}$.

TUNEL assay. SGC7901 and SGC7901/VCR cells were digested and plated on glass coverslips in 24-well plates. Subsequently, the drug was added to the corresponding wells of each group with the final volume of $2.2 \mathrm{ml}$. Cells were cultured at $37^{\circ} \mathrm{C}$ in a humidified atmosphere containing $5 \%$ $\mathrm{CO}_{2}$ for $72 \mathrm{~h}$ and then fixed with $4 \%$ paraformaldehyde for $1 \mathrm{~h}$. Endogenous peroxidase was blocked by $3 \% \mathrm{H}_{2} \mathrm{O}_{2}$ in methanol for $10 \mathrm{~min}$. Subsequently, cells were rinsed with PBS and incubated with permeabilization solution for $2 \mathrm{~min}$ on ice. The coverslips were washed twice with PBS and then $50 \mu \mathrm{l}$ of the TUNEL reaction mixture (Roche, Boulder, CO, USA) were added to the cells. Finally, the coverslips were stained with DAB for $10 \mathrm{~min}$, washed twice with PBS and were observed under a microscope. Labeled nuclei and the total number of cells were counted in at least five different fields.

$R T-P C R$. RT-PCR was performed for semiquantitative detection of the mRNA expression of $M D R 1, \mathrm{Bax}, \mathrm{Bcl}-2$, XIAP and caspase-3 in SGC-7901 and SGC-7901/VCR cells using the Access RT-PCR system (Promega Co., Madison, WI, USA). The primers for the above genes were synthesized (Table I). Total RNA was isolated using Tripure reagent (Roche Diagnostics, Mannheim, Germany) using standard methods. The total volume of the RT-PCR reaction system was $25 \mu \mathrm{l}$, including $0.2 \mathrm{mmol} / 1 \mathrm{dNTP}, 1 \mathrm{mmol} / 1$ of each primer, $1 \mathrm{mmol} / 1 \mathrm{MgSO}_{4}, 0.1 \mathrm{U} / \mathrm{ml}$ AMV transcriptase, $0.1 \mathrm{U} / \mathrm{ml} \mathrm{Tf1}$ DNA polymerase, $1 \mathrm{X}$ reaction buffer and $100 \mathrm{ng}$ of RNA template. The amplification conditions were $48^{\circ} \mathrm{C}$ for $45 \mathrm{~min}$ for cDNA synthesis and 40 cycles $\left(94^{\circ} \mathrm{C}, 30 \mathrm{sec} ; 60^{\circ} \mathrm{C}, 30 \mathrm{sec}\right.$; $72^{\circ} \mathrm{C}, 1 \mathrm{~min}$ ) for PCR (Perkin-Elmer Cetus, Norwalk, CA, USA). The amplified products were electrophoresed on a $2 \%$ agarose gel and visualized after ethidium bromide staining of the gel. $\beta$-actin was used as an internal control.

Immunohistochemical staining. The protein expression of P-glycoprotein (P-gp), Bax, Bcl-2, caspase-3 and XIAP in SGC-7901 and SGC-7901/VCR cells was detected by immunohistochemical assay. Briefly, cells were plated in 24-well plates ( $3 \times 10^{4}$ cells/well) and cultured for $72 \mathrm{~h}$. The cells were washed and fixed with $70 \%$ ethanol and then $0.3 \% \mathrm{H}_{2} \mathrm{O}_{2}$-methanol solution was added to each well, and the plates were placed in a $25^{\circ} \mathrm{C}$ incubator for $30 \mathrm{~min}$ to inhibit the activity of endogenous peroxidase. Subsequently, the cells were incubated with the primary antibodies for P-gp, Bax, Bcl-2, caspase-3 or XIAP (Beijing ZhongShan Biotechnology Co., Beijing, China) at $4^{\circ} \mathrm{C}$ overnight. After a thorough wash with PBS containing $0.1 \%$ Triton $\mathrm{X}-100$, the cells were incubated with
Table I. Primers for RT-PCR analysis.

\begin{tabular}{lc}
\hline Genes & \multicolumn{2}{c}{ Primers } \\
\hline MDR1 & \\
Sense & 5'-CTCGAGGAATCAGCATTCAG-3' \\
Antisense & 5'-AGATCTCTTTGAGCTTGGAAGAGC-3' \\
Bax & \\
Sense & 5'-CTGACATGTTTTCTGACGGC-3' \\
Antisense & 5'-TCAGCCCATCTTCTTCCAGA-3' \\
Bcl-2 & \\
Sense & 5'-ACACTGTTAAGCATGTGCCG-3' \\
Antisense & 5'-CCAGCTCATCTCACCTCACA-3' \\
XIAP & \\
Sense & 5'-TGGCACGAGCAGGGTTTCTTT-3' \\
Antisense & 5'-TGGCACGAGCAGGGTTTCTTT-3' \\
Caspase-3 & \\
Sense & 5'-AAGCGAATCAATGGACTCTG-3' \\
Antisense & 5'-GACTTCTACAACGATCCCCTC-3' \\
3-actin & 5'-CCACGAAACTACCTTCAACTCC-3' \\
Sense & 5'-ACTCGTCATACTCCTGCTTGCT-3' \\
Antisense & \\
\hline
\end{tabular}

a secondary antibody (Beijing ZhongShan Biotechnology Co.) for $30 \mathrm{~min}$ at $25^{\circ} \mathrm{C}$. Finally, cells were incubated for $15 \mathrm{~min}$ with an avidin-biotin enzyme reagent (Beijing ZhongShan Biotechnology Co.). The staining was performed by adding a 3,3-diaminobenzidine $/ \mathrm{H}_{2} \mathrm{O}_{2}$ solution in each well under an inverted microscope. The staining reaction was terminated by adding PBS to each well. The figure was captured and analyzed by IPP5.0 (Media Cybernetics, Rockville, MD, USA).

Tumor xenografts in nude mice. Twenty-four nude mice at four weeks of age were maintained in a pathogen-free animal facility in accordance with the local Ethics Committee of The Third Military Medical University. Mice were divided into four groups: the VCR group, the LY294002 group, the VCR + LY294002 group, and the control group. SGC7901 and SGC7901/VCR cells were digested and injected subcutaneously into nude mice to induce gastric tumor xenografts. After 10 days, mice were treated with VCR (40-50 mg/ $\mathrm{kg}$ body weight) and/or LY294002 (15-20 mg/kg body weight) once per day intraperitoneally for one week. The mice were sacrificed when established tumors in the control group reached $\sim 1 \mathrm{~cm}^{3}$. Then, the tumor was stripped out and blood, fat, necrotic tissues and non-tumor ingredients were removed. Tumor was weighed (tumor weight in grams) and tumor inhibition rate was calculated. Tumor inhibition rate $=$ (average tumor weight of control group - the average tumor weight of experimental group)/average tumor weight of control group x $100 \%$.

Statistical analysis. The data are expressed as the means \pm standard deviation (SD). Statistical analysis of the data was performed using SPSS 17.0 (SPSS, Chicago, IL, USA). Statistical significance was determined using ANOVA followed by Student's t-test. A value of $\mathrm{P}<0.05$ was considered to indicate a statistically significant difference. 

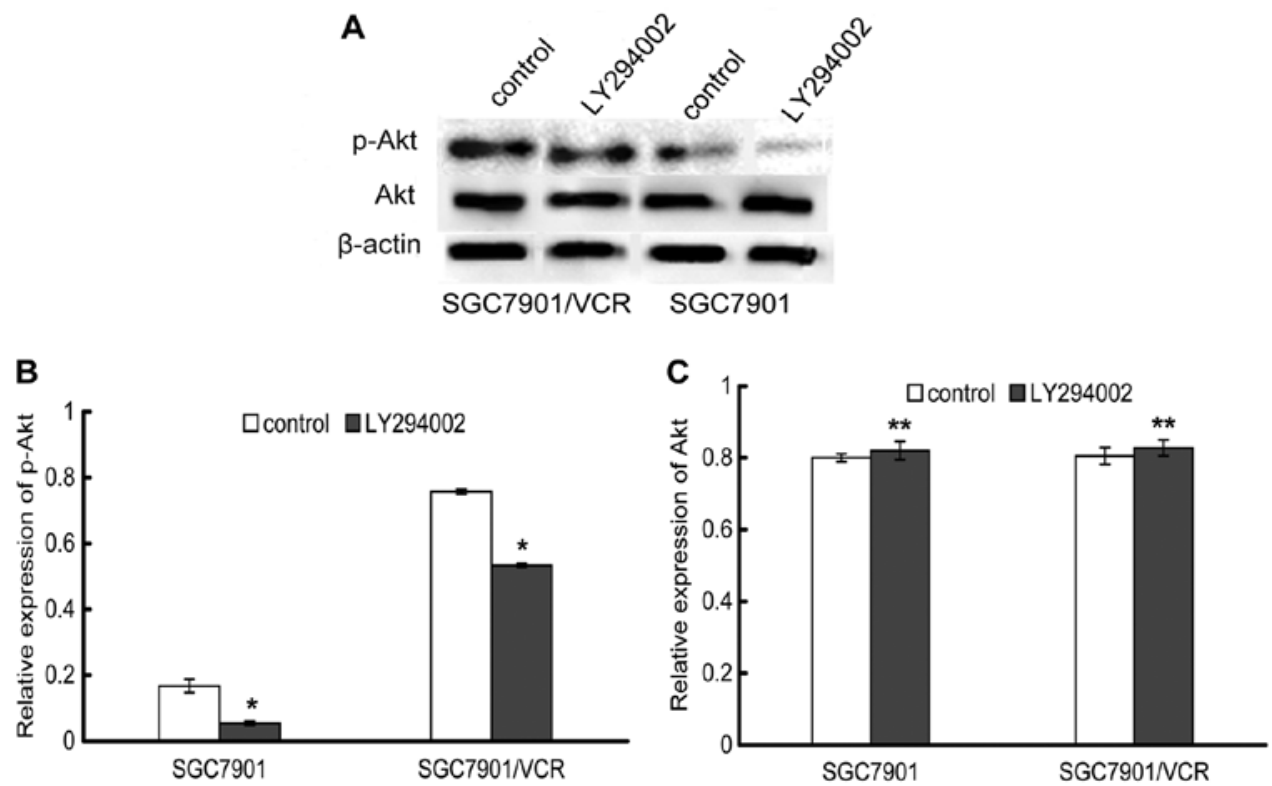

Figure 1. Western blot analysis for the inhibition of the PI3K/Akt pathway by LY294002. Cells were treated with LY294002 or not, and the expression of Akt and p-Akt proteins was assessed by western blot analysis. $\beta$-actin was used as an internal control (A). Density of each band was quantified, and the ratio of the band with each treatment vs. $\beta$-actin was calculated and presented as the relative expression (B and C). Each value represents the mean \pm SD of three independent experiments. "P<0.01 vs. control; ** $\mathrm{P}>0.05$ vs. control.
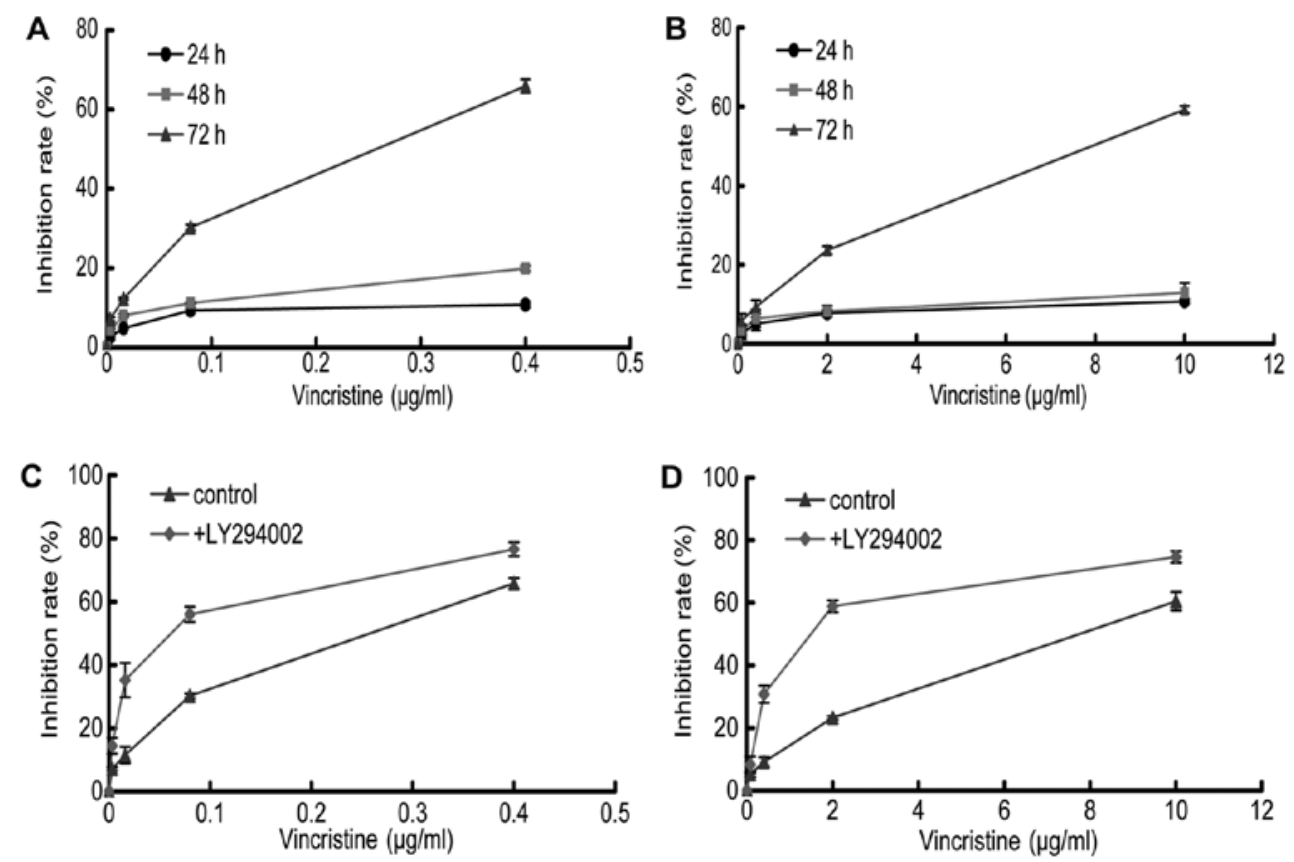

Figure 2. The effect of LY294002 on the susceptibility of gastric cancer cells to vincristine (VCR). Human gastric cancer cell lines SGC7901 (A) and SGC7901/VCR (B) were treated with various concentrations of VCR for different time points. SGC7901 (C) and SGC7901/VCR cells (D) were treated with LY294002 and/or VCR for $72 \mathrm{~h}$. Cell viability was measured for analysis of $\mathrm{IC}_{50}$ using MTT assay. Data are means \pm SD from four independent experiments.

\section{Results}

Inhibition of the PI3K/Akt signaling pathway by LY294002. To explore the possible role of the PI3K/Akt pathway in SGC7901 and SGC7901/VCR cells and to evaluate the influence of LY294002 on PI3K activity, phosphorylated Akt and total Akt were examined by western blot analysis. As expected, the phospho-Akt level in SGC7901/VCR cells was higher than that in SGC7901 cells, suggesting a higher PI3K/Akt activity in the resistant SGC7901/VCR cells. Treatment of these two cell lines with $20 \mu \mathrm{mol} / 1$ of LY294002 caused inactivation of Akt, as seen by a significant decrease in Akt phosphorylation. However, LY294002 did not inhibit the expression of total Akt, thereby demonstrating that LY294002 inhibits the activity of Akt and not its expression (Fig. 1).

LY294002 enhances the sensitivity of gastric cancer cells to $V C R$. The MTT assay was performed to evaluate the effect of 
Table II. Intracellular concentrations of VCR in cells.

\begin{tabular}{|c|c|c|c|c|}
\hline \multirow{2}{*}{$\begin{array}{l}\text { Groups } \\
\text { Time point }\end{array}$} & \multicolumn{2}{|c|}{ SGC7901 } & \multicolumn{2}{|c|}{ SGC7901/VCR } \\
\hline & $3 \mathrm{~h}$ & $6 \mathrm{~h}$ & $3 \mathrm{~h}$ & $6 \mathrm{~h}$ \\
\hline VCR & $6.87 \pm 0.06$ & $7.58 \pm 0.12$ & $0.00 \pm 0.00$ & $0.00 \pm 0.00$ \\
\hline LY294002 + VCR & $13.47 \pm 0.14^{\mathrm{a}}$ & $15.56 \pm 0.14^{\mathrm{a}}$ & $0.00 \pm 0.00$ & $0.17 \pm 0.01^{\mathrm{a}}$ \\
\hline
\end{tabular}

Intracellular concentrations of VCR in cells were determined by the high performance liquid chromatography. ${ }^{\mathrm{a}} \mathrm{P}<0.01 \mathrm{vs}$. VCR. VCR, vincristine.

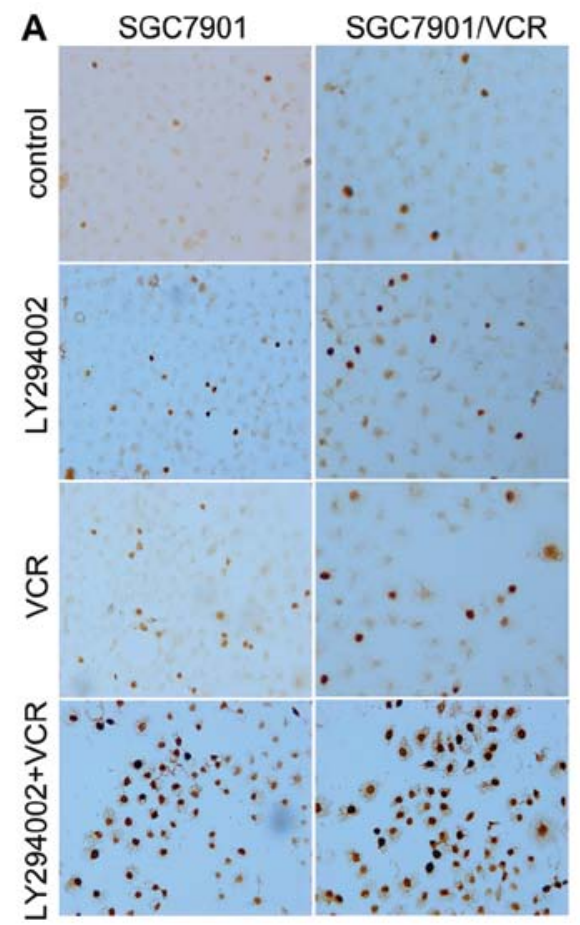

\section{B}

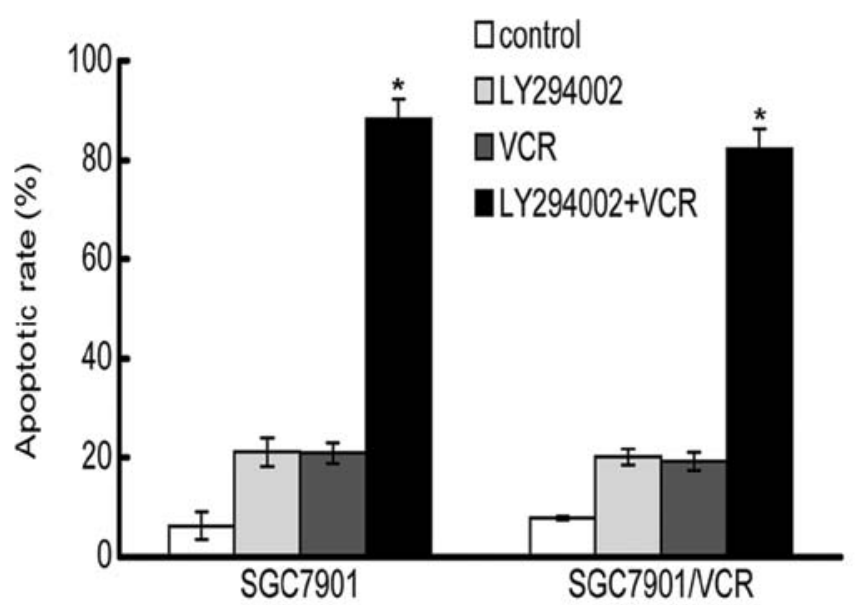

Figure 3. Apoptosis induction in SGC7901 and SGC7901/VCR cells. Cells were treated with LY294002 and/or vincristine (VCR) and were evaluated by TUNEL staining. TUNEL-positive cells were stained dark with a condensed nucleus, visualized by a microscope (A). Summary graph of the counting results from TUNEL staining (B). "P $<0.05$ vs. control, LY294002, VCR.

LY294002 and/or VCR on the proliferation of SGC7901 and SGC7901/VCR cells. The results showed that VCR was cytotoxic to the two cell lines in a dose and time-dependent manner at $72 \mathrm{~h}$, with an $\mathrm{IC}_{50}$ of $0.2 \pm 0.03 \mu \mathrm{g} / \mathrm{ml}$ and $8.09 \pm 0.6 \mu \mathrm{g} / \mathrm{ml}$ for the SGC7901 and SGC7901/VCR cells, respectively (Fig. 2). The resistance index (ratio of $\mathrm{IC}_{50}$ for resistant and parental cells) was $\sim 40.45$, demonstrating that the SGC7901/VCR cells were highly resistant to VCR. The growth inhibitory effect of VCR was significantly reduced in SGC7901/VCR cells compared with their parental cells. Subsequently, we combined LY294002 $(20 \mu \mathrm{mol} / \mathrm{l})$ with varying concentrations of VCR to test whether this combination could further inhibit growth of gastric cancer cells. The addition of LY294002 significantly increased the potency of VCR in these two cell lines, and the $\mathrm{IC}_{50}$ decreased further to $0.05 \pm 0.006 \mu \mathrm{g} / \mathrm{ml}$ and $1.70 \pm 0.20 \mu \mathrm{g} / \mathrm{ml}$, respectively (Fig. 2). We then used the combination index method to determine the exact interactions between LY294002 and VCR. The combination index for the two drugs was $<1$, indicating their synergistic effect.
LY294002 increases the intracellular concentrations of $V C R$ in gastric cells. To investigate whether the inhibition of the PI3K/Akt pathway by LY294002 could result in increased accumulation of VCR in gastric cancer cell lines, we performed HPLC to determine the intracellular concentrations of VCR in these cells, either pretreated with LY294002 or not. The results showed that the VCR concentration in SGC7901 cells treated with LY294002 was twice as much as that in SGC7901 cells without treatment with LY294002 (Table II). In SGC7901/VCR cells, the concentration of VCR was undetectable without treatment with LY294002, thereby demonstrating a high resistance of the cells to VCR. However, the concentration of VCR was detected $6 \mathrm{~h}$ after LY294002 treatment, suggesting that LY294002 could increase the accumulation of VCR in resistant gastric cancer cells (Table II).

LY294002 enhances VCR-induced apoptosis. Apoptosis was determined by the detection of DNA strand breaks using TUNEL staining. SGC7901 and SGC7901/VCR cells that 
A
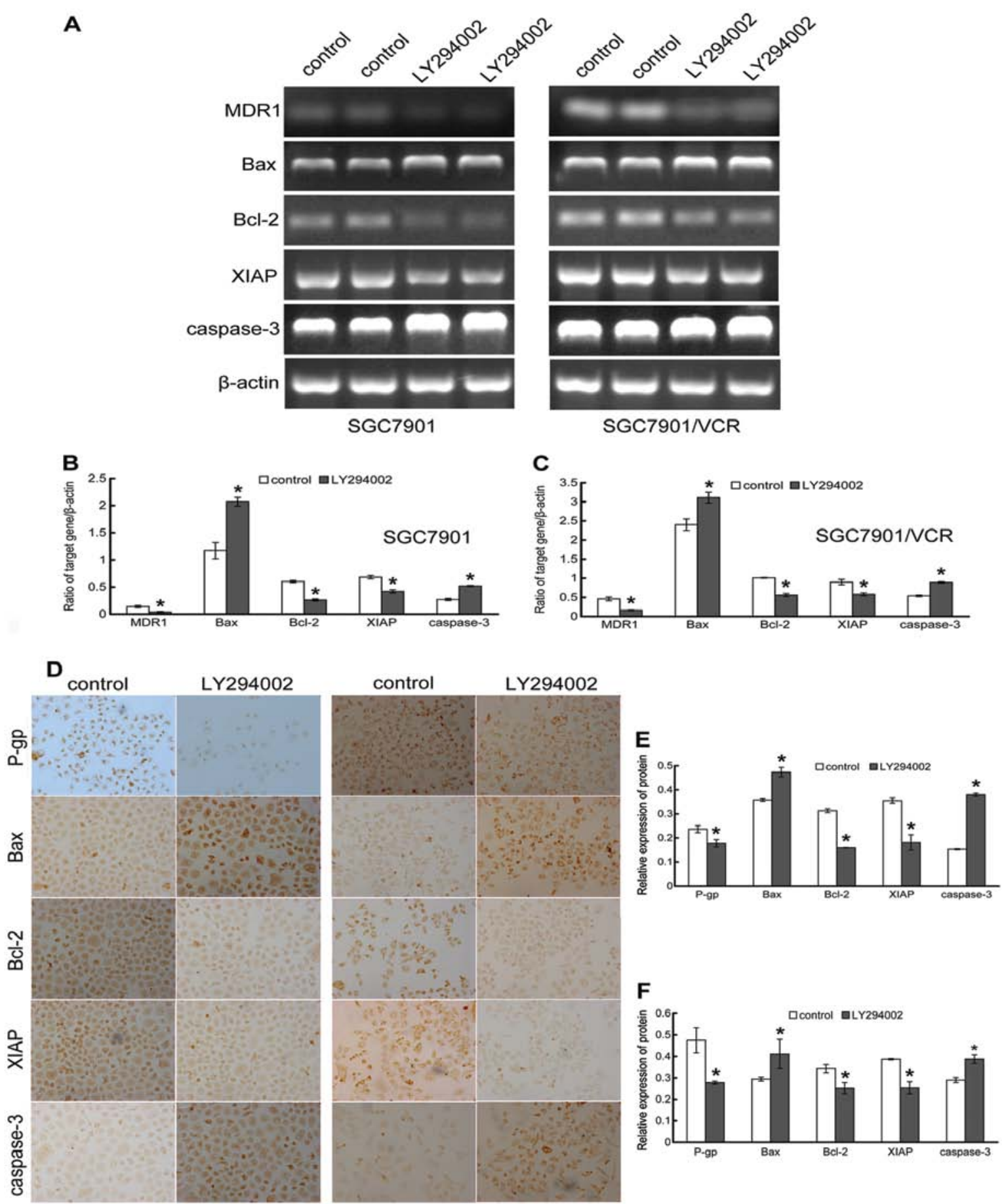

Figure 4. The effect of LY294002 on the expression levels of MDR1/P-gp, Bax, Bcl-2, XIAP and caspase-3 in SGC7901 and SGC7901/VCR cells. The mRNA expression was evaluated in SGC7901 and SGC7901/VCR cells treated with LY294002 using semi-quantitative RT-PCR. $\beta$-actin was used as a loading control. The density of each band was quantified, and gene levels were calculated relative to the amount of $\beta$-actin gene (A-C). The protein expression levels in SGC7901 and SGC7901/VCR cells were detected by immunocytochemistry (D). The mean density was calculated using IPP5.0 (E and F). ${ }^{*} \mathrm{P}<0.01$ vs. control.

were treated with either LY294002 or VCR alone showed fewer apoptotic cells compared with the control. However, following treatment with LY294002 and VCR, a significant increase in the apoptotic cells was observed, suggesting that LY294002 could markedly enhance VCR-induced apoptosis. The results from the cell counts were expressed as the percentage of apoptotic cells among the total cells observed in each microscopic field, and the resulting apoptotic indices showed a significant increase in cells treated with the combination of LY294002 and VCR $(\mathrm{P}<0.05)$ (Fig. 3).

Effect of LY294002 on the expression of MDR1/P-gp and apoptosis-related factors. To further illustrate the mechanism by which inhibition of PI3K/Akt enhances the chemosensitivity of resistant gastric cancer cells to VCR, the mRNA [semiquantitative reverse transcription PCR (RT-PCR)] and 
Table III. Average weight of tumor and inhibition rate of tumor in each group.

\begin{tabular}{lcccc}
\hline Cells & Groups & No. & Tumor size $(\mathrm{g})$ & Inhibition rate $(\%)$ \\
\hline SGC7901 & Control & 3 & $1.219 \pm 0.041$ & - \\
& LY294002 & 3 & $1.111 \pm 0.102$ & $8.862 \pm 8.361$ \\
& VCR & 3 & $0.809 \pm 0.043$ & $33.590 \pm 3.544$ \\
SGC7901/VCR & LY294002+VCR & 3 & $0.289 \pm 0.027^{\mathrm{a}}$ & $76.279 \pm 2.194^{\mathrm{a}}$ \\
& Control & 3 & $0.795 \pm 0.061$ & - \\
& LY294002 & 3 & $0.727 \pm 0.076$ & $8.489 \pm 0.768$ \\
& VCR & 3 & $0.674 \pm 0.009$ & $15.160 \pm 1.082$ \\
& LY294002+VCR & 3 & $0.283 \pm 0.042^{\mathrm{a}}$ & $64.348 \pm 5.308^{\mathrm{a}}$ \\
\hline
\end{tabular}

${ }^{\mathrm{a} P}<0.05$ vs. LY294002, VCR. VCR, vincristine.

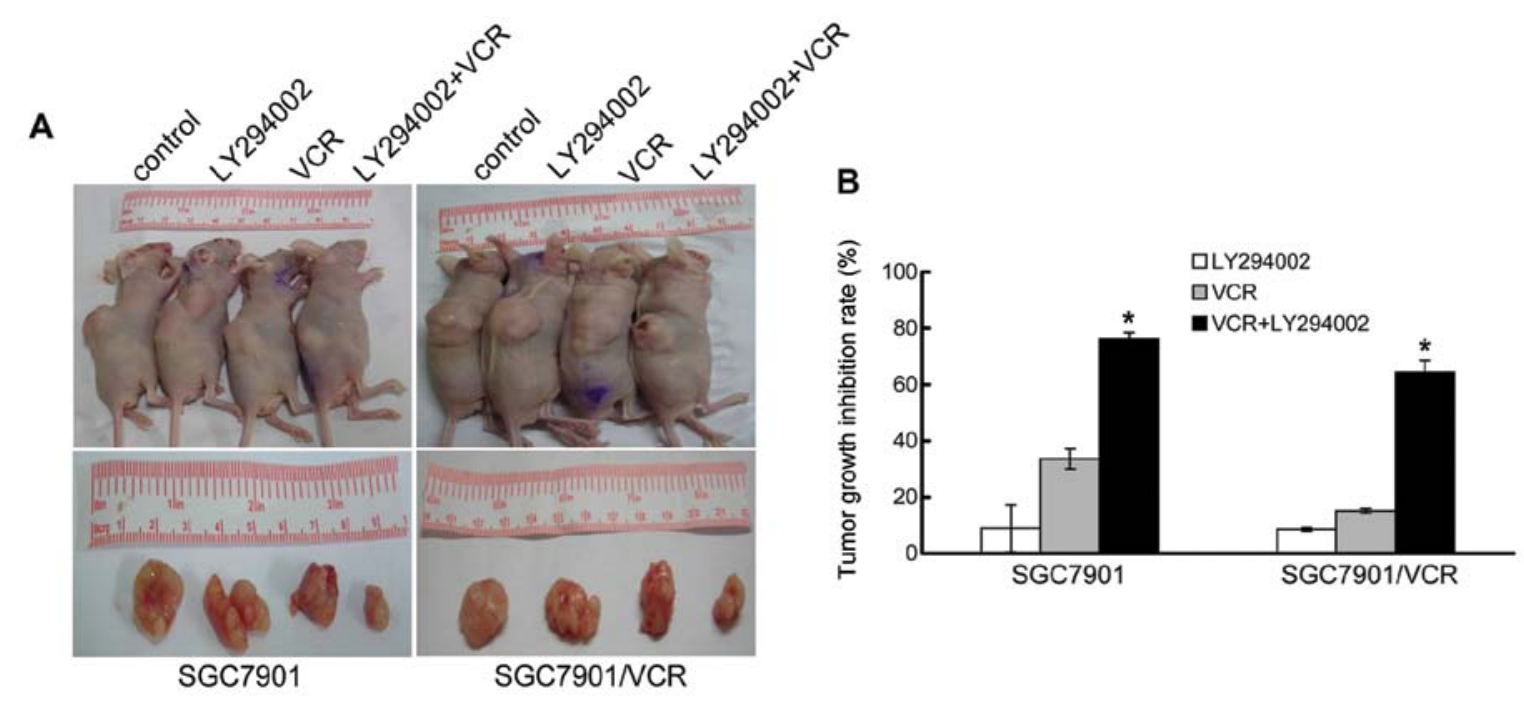

Figure 5. In vivo effect of LY294002 on vincristine (VCR)-induced suppression of tumor growth. Tumor xenografts in nude mice bearing SGC7901 cells and SGC7901/VCR cells were divided into four groups and treated with different drugs (A). Tumor growth inhibition rate was obtained using the formula mentioned in Materials and methods (B). "P<0.05 vs. LY294002, VCR.

protein (immunohistochemical staining) expression of the $M D R 1 / \mathrm{P}-\mathrm{gp}$ and apoptosis-related factors was analyzed. Our results showed that the expression levels of $M D R l$ were higher in SGC7901/VCR cells than that in SGC7901 cells, suggesting an increased activity of the drug pump in these resistant cells (Fig. 4), which was in accordance with the lower concentration of VCR in SGC7901/VCR cells. Inhibition of the PI3K/Akt pathway by LY294002 significantly decreased the expression of $M D R 1$ at both the mRNA and protein levels, suggesting transcriptional regulation of $M D R l$ by $\mathrm{PI} 3 \mathrm{~K} / \mathrm{Akt}$ in gastric cancer cells (Fig. 4).

Our previous results showed that inhibition of PI3K/Akt by LY294002 could significantly enhance VCR-induced apoptosis. Given that the PI3K/Akt activity was actively involved in the apoptotic process, we hypothesized that the PI3K activity may modulate the expression patterns of one or more apoptosis-related factors. As anticipated, LY294002 treatment resulted in the downregulation of anti-apoptotic factors Bcl-2 and XIAP, and upregulation of pro-apoptotic factors Bax and caspase- 3 at both the mRNA and protein levels in the cells
(Fig. 4). Therefore, inhibition of the PI3K/Akt pathway may enhance VCR-induced apoptosis, partly by regulating the transcription of these apoptosis-related factors.

In vivo effect of LY294002 on tumor growth. Next, we evaluated the effects of LY294002, VCR and the combination of these two drugs on the growth of SGC7901 and SGC7901/VCR cells in vivo, which were injected subcutaneously in nude mice. In both cell lines, treatment with either LY294002 or VCR alone resulted in a slight decrease in tumor growth compared to control untreated mice (Fig. 5). The results showed that LY294002 had almost no effect on the inhibition of tumor growth in vivo, consistent with the in vitro results. The tumor growth inhibition following VCR treatment in SGC7901/VCR cells was less than that in SGC7901 cells, indicating significant resistance to VCR. However, treatment with LY294002 and VCR together caused a significant inhibition of tumor growth of SGC7901 and SGC7901/VCR cells in vivo compared to LY294002 or VCR alone $(\mathrm{P}<0.05)$ (Fig. 5, Table III). 


\section{Discussion}

PI3K and its downstream signaling protein Akt have been implicated in the regulation of major responses to extracellular growth stimulation, including cell proliferation, development, differentiation, cell cycle and apoptosis $(19,20)$. Constitutive activation of the PI3K signaling pathway is commonly observed in various types of cancer $(13,21)$ and is considered to contribute to tumorigenesis by simultaneously promoting proliferation and inhibiting apoptosis (22). Excessive activation of PI3K/Akt is found in gastric cancer and is positively correlated to tumor stage (23); it is also associated with chemoresistance in multiple cancer cells including gastric cancer (24), suggesting a close association with carcinogenesis, progression and MDR in gastric cancer. Therefore, inhibition of PI3K/Akt signaling can serve as a potentially useful approach for the treatment of gastric cancer.

In the present study, we investigated whether LY294002, by inhibition of PI3K/Akt, could increase the chemosensitivity of human gastric cancer cells SGC7901 and the related vincristine (VCR)-resistant cells SGC7901/VCR, to VCR. When the two cell lines were exposed to VCR, cell proliferation was inhibited in a dose- and time-dependent manner. The $\mathrm{IC}_{50}$ of SGC7901/VCR cells exposed to VCR was clearly higher than that of SGC7901 cells, with a resistance index of approximately 40.45, demonstrating that SGC7901/VCR cells are chemoresistant to VCR. Subsequently, when cells were treated with $20 \mu \mathrm{M}$ LY294002 in combination with various concentrations of VCR, the rate of growth inhibition increased significantly in both SGC7901 and SGC7901/VCR cells, with an approximately 4and 4.76-fold reduction in $\mathrm{IC}_{50}$, respectively. However, the $\mathrm{IC}_{50}$ value of LY294002 in both SGC7901 and SGC7901/VCR cells was similar, therefore LY294002 alone would be expected to have similar effects in these two cell lines. LY294002 not only enhanced chemosensitivity to VCR but also exerted synergetic inhibition of proliferation in SGC7901 and SGC7901/VCR cells. Furthermore, we investigated the effects of LY294002 on tumor growth in tumor-bearing nude mice. As seen in vitro, LY294002 combined with VCR could significantly inhibit the growth of tumors in vivo, and the inhibition rate was significantly higher than LY294002 or VCR alone. Akt is a key regulator of cell survival and apoptosis, and increased Akt phosphorylation is a surrogate for high PI3K/Akt activity. The results showed that the resistant SGC7901/VCR cells presented higher PI3K/Akt activity than SGC7901 cells using western blotting, which is in accordance with the close relationship between PI3K signaling and drug resistance phenotype. Following treatment with LY294002, the phospho-Akt expression level in these two cell lines decreased significantly, suggesting that the drug is highly potent in inhibiting the PI3K/Akt signaling pathway. In addition, we found that there was no alteration of total Akt in response to LY294002, indicating that downregulation of Akt phosphorylation was independent of downregulation of the total Akt in these cells. These results indicated that PI3K/Akt inhibition by LY294002 could suppress tumor cell proliferation and enhance chemosensitivity to VCR in human gastric cancer cells.

Several mechanisms may be implicated in MDR, an important one being the prevention of intracellular accumulation of anti-tumor drugs by the expression of transport proteins that pump drugs out of cancer cells. The MDRl gene product P-gp (also known as ABCB1) is one of the most thoroughly studied transport proteins (25). Previous studies indicated that the activation of the PI3K/Akt signaling pathway was closely associated with upregulation of MDR1 expression $(26,27)$. Similarly, our study found that high PI3K/Akt activity resulted in increased expression of MDR1 or P-gp in the resistant SGC7901/VCR cells when compared to SGC7901 cells, leading to a decline in VCR accumulation in SGC7901/VCR cells in the absence of LY294002. Therefore, we inferred that LY294002 treatment may interfere with the intracellular trafficking of VCR by interrupting with P-gp activity. Our results showed that an increase in VCR accumulation was observed in SGC7901/VCR cells treated with LY294002, most likely due to a significant downregulation of P-gp after treatment with LY294002 in these cells. However, a great deal remains to be understood about how P-gp expression is regulated. Previous studies had demonstrated that $M D R 1$ expression was driven by the NF- $\kappa \mathrm{B}$ pathway, using a common agonist known as the phorbol ester 12-O-tetradecanoyl-13-acetate (TPA) $(28,29)$. Crosstalk between the PI3K/Akt pathway and NF- $\kappa \mathrm{B}$ has been demonstrated; NF- $\kappa$ B expression can be downregulated by treatment with LY294002 (26,30), indicating that downregulation of $M D R I$ expression is mediated by inhibition of the PI3K/Akt pathway. Even so, the relationship between signal transduction pathways and $\mathrm{P}$-gp expression is a complex process involving more than a single pathway, of which we have highlighted regulation by the PI3K/Akt pathway. Additionally, regulation of P-gp phosphorylation by the Akt kinase or direct interaction of P-gp with LY294002 cannot be excluded (27). Of note, the fact that the presence of both LY294002 and VCR in SGC7901/VCR cells induced detectable drug accumulation only after $6 \mathrm{~h}$ indicates that the effect of LY294002 was relatively delayed. Thus, we can infer that the mechanism of LY294002 action involves downregulation of P-gp expression rather than direct interaction with the drug binding site of P-gp.

The susceptibility of cancer cells to apoptosis induced by chemotherapeutic drugs depends on the balance between pro-apoptotic and anti-apoptotic signals. Altered cellular responses to apoptosis and induction of anti-apoptotic proteins are thought to play an important role in drug resistance in cancer cells. PI3K/Akt plays a vital role in mediating survival signals, contributing to the inhibition of apoptosis and therapeutic resistance through multiple mechanisms $(31,32)$. Therefore, the inhibition of the PI3K/Akt pathway may promote chemotherapeutic drug-induced apoptosis and consequently enhance the chemosensitivity of various types of cancer. Our results further confirmed that LY294002 combined with VCR could significantly induce higher apoptosis levels in SGC7901 and SGC7901/VCR cells than the effects of LY294002 or VCR alone. There are two major pathways that initiate apoptosis and eventually lead to activation of caspases: the death receptor (extrinsic) pathway and the mitochondrial (intrinsic) pathway $(33,34)$. The anti-cancer drugs mainly initiate the intrinsic pathway, which is engaged by the release of apoptogenic factors such as cytochrome $c$ into the cytosol that further triggers caspase-3 activation $(35,36)$. The mitochondria-mediated apoptosis is mainly regulated by the Bcl-2 family of proteins, which consists of 
anti-apoptotic proteins Bcl-2 and Bcl-xL, as well as a number of pro-apoptotic proteins such as Bax, Bid and Bim (36-38). In addition, XIAP is a member of the inhibitor of apoptosis protein (IAP) family, the only member capable of blocking active caspases, which can inhibit apoptosis by binding and thereby inactivating certain caspases including initiator caspase- 9 and the effector caspase-3 (39). In the present study, we showed that in SGC7901 and SGC7901/VCR cells, the PI3K/Akt inhibitor LY294002 could downregulate the expression of Bcl-2 and XIAP, and upregulate the expression of Bax and caspase-3. Downregulation of Bcl-2 in turn allows conformational changes in the Bax protein and subsequent translocation to mitochondria, leading to the release of cytochrome $c$ into the cytoplasm. Downregulation of XIAP along with the release of cytochrome $c$ results in a sequential activation of caspase- 9 and caspase-3, and initiates the apoptotic cascade (40). Our results indicated that the expression levels of these apoptotic factors that are regulated by inhibition of PI3K/Akt are not merely dependent on caspase activity or direct interactions with some regulatory factors; there is also a significant regulation of their transcriptional levels. It is known that among the various functions of the PI3K/Akt pathway, Akt inhibits apoptosis either directly by phosphorylating apoptosis-signaling molecules or indirectly by modulating the activity of transcription factors (41). The cross-talk between the PI3K/Akt pathway and the NF- $\kappa \mathrm{B}$ pathway results in Akt-mediated activation of the transcription factors CREB (c-AMP response element binding protein) or I $\kappa$ B kinases (IKKs) that further activate NF- $\kappa$ B to exert an anti-apoptotic effect $(24,40,42,43)$. Inhibition of $N F-\kappa B$ may lead to a reduced transcription of target genes such as Bcl-2 and XIAP $(30,40)$, as was shown in our present results. Since caspase- 3 is at the crossroad of extrinsic and intrinsic apoptosis pathways (40), inhibition of PI3K/Akt by LY294002 can simultaneously affect these two pathways, consistent with a previous report implicating the PI3K/Akt pathway in apoptosis mediated by both the death receptor and the mitochondrial pathway (44). A previous report indicated that P-gp may counteract apoptosis by transporting a key caspase out of the cell or inhibiting caspase activity, and in turn be cleaved in a caspase-dependent manner during apoptosis (45); this demonstrates the complexity of the apoptosis and drug resistance signaling network. There certainly may be other pathways or regulatory factors involved in the complicated mechanisms of MDR, and this will be the direction of our future research focus. Presently, we have demonstrated that inhibition of the PI3K/Akt pathway by LY294002 could significantly enhance VCR-induced apoptosis in gastric cancer cells, predominantly through downregulation of anti-apoptotic factors and upregulation of apoptotic factors.

A recent study provided key evidence that the use of LY294002 in cancer therapy can be detrimental when the PI3K signaling pathway is inhibited prior to anticancer drug administration (46). This may be because the inhibition of PI3K/ Akt decreases the proliferation of various cancer cell lines by preventing cell cycle progression, particularly at the G1-phase; since several chemotherapeutic agents depend mainly on cell proliferation for their cytotoxic activities, inhibition of PI3K may lead to decreased susceptibility to cell cycle-dependent chemotherapy $(46,47)$. Therefore, the timing of administering
LY294002 is critical in the context of chemotherapy, and using LY294002 after chemotherapeutic agents may act in a synergistic manner to enhance chemosensitivity of cancer cells. Moreover, the inhibition of PI3K/Akt in different cancer cell lines in relation to diverse chemotherapeutic agents, may exert different effects $(24,32)$. Therefore, it is necessary to illustrate the complicated interactions between signaling pathways and $\mathrm{MDR}$, in order to conduct a rational combination of various chemotherapeutic agents in the treatment of cancer.

In conclusion, our results demonstrate that the PI3K/Akt inhibitor LY294002 can enhance chemosensitivity of VCR in human gastric cancer cells by inactivation of the PI3K/Akt signaling pathway. Therefore, our results indicate that modulation of the PI3K/Akt pathway may provide a new strategy to improve current therapeutic regimens, and this preclinical evaluation of a rational combination of LY294002 and VCR in relevant in vitro and in vivo models of gastric cancer also provides a molecular basis for the new design of combination treatments for gastric cancer.

\section{Acknowledgements}

The authors thank Professor Shiming Yang (Department of Gastroenterology, Xinqiao Hospital, Third Military Medical University) and the technician Yuyun Wu (Department of Gastroenterology, Xinqiao Hospital, Third Military Medical University) for their excellent technical support.

\section{References}

1. Ferlay J, Shin HR, Bray F, Forman D, Mathers C and Parkin DM: Estimates of worldwide burden of cancer in 2008: GLOBOCAN 2008. Int J Cancer 127: 2893-2917, 2010.

2. Plummer M, Franceschi S and Muñoz N: Epidemiology of gastric cancer. IARC Sci Publ 157: 311-326, 2004.

3. Catalano V, Labianca R, Beretta GD, Gatta G, de Braud F and Van Cutsem E: Gastric cancer. Crit Rev Oncol Hematol 71: 127-164, 2009.

4. Ozeben T: Mechanisms and strategies to overcome multiple drug resistance in cancer. FEBS Lett 580: 2903-2909, 2006.

5. Gillet JP and Gottesman MM: Mechanisms of multidrug resistance in cancer. Methods Mol Biol 596: 47-76, 2010.

6. Gillet JP, Efferth T and Remacle J: Chemotherapy-induced resistance by ATP-binding cassette transporter genes. Biochim Biophys Acta 1775: 237-262, 2007.

7. Callaghan R, Crowley E, Potter S and Kerr ID: P-glycoprotein: so many ways to turn it on. J Clin Pharmacol 48: 365-378, 2008.

8. Martin LP, Hamilton TC and Schilder RJ: Platinum resistance: the role of DNA repair pathways. Clin Cancer Res 14: 1291-1295, 2008.

9. Sarkaria JN, Kitange GJ, James CD, Plummer R, Calvert H, Weller M and Wick W: Mechanisms of chemoresistance to alkylating agents in malignant glioma. Clin Cancer Res 14: 2900-2908, 2008.

10. Rodriguez-Nieto S and Zhivotovsky B: Role of alterations in the apoptotic machinery in sensitivity of cancer cells to treatment. Curr Pharm Des 12: 4411-4425, 2006.

11. Viktorsson K, Lewensohn R and Zhivotovsky B: Apoptotic pathways and therapy resistance in human malignancies. Adv Cancer Res 94: 143-196, 2005.

12. Kim D, Dan HC, Park S, Yang L, Liu Q, Kaneko S, Ning J, He L, Yang H, Sun M, Nicosia SV and Cheng JQ: AKT/PKB signaling mechanisms in cancer and chemoresistance. Front Biosci 10: 975-987, 2005.

13. Liu P, ChenG H, Roberts TM and Zhao JJ: Targeting the phosphoinositide 3-kinase pathway in cancer. Nat Rev Drug Discov 8: 627-644, 2009.

14. Courtney KD, Corcoran RB and Engelman JA: The PI3K pathway as drug target in human cancer. J Clin Oncol 28: 1075-1083, 2010 
15. Fresno Vara JA, Casado E, de Castro J, Cejas P, Belda-Iniesta C and González-Barón M: PI3K/Akt signalling pathway and cancer. Cancer Treat Rev 30: 193-204, 2004.

16. Kunnimalaiyaan M, Ndiaye M and Chen H: Apoptosis-mediated medullary thyroid cancer growth suppression by the PI3K inhibitor LY294002. Surgery 140: 1009-1014, 2006.

17. Liu P, Xu B, Li J and Lu H: LY294002 inhibits leukemia cell invasion and migration through early growth response gene 1 induction independent of phosphatidylinositol 3-kinase-Akt pathway. Biochem Biophys Res Commun 377: 187-190, 2008

18. Imai Y, Yoshimori M, Fukuda K, Yamagishi H and Ueda Y: The PI3K/Akt inhibitor LY294002 reverses BCRP-mediated drug resistance without affecting BCRP translocation. Oncol Rep 27: $1703-1709,2012$

19. Wu D, Tao J, Xu B, Qing W, Li P, Lu Q and Zhang W: Phosphatidylinositol 3-kinase inhibitor LY294002 suppresses proliferation and sensitizes doxorubicin chemotherapy in bladder cancer cells. Urol Int 87: 105-113, 2011.

20. Gelman AE, LaRosa DF, Zhang J, Walsh PT, Choi Y, Sunyer JO and Turka LA: The adaptor molecule MyD88 activates PI-3 kinase signaling in $\mathrm{CD}^{+} \mathrm{T}$ cells and enables $\mathrm{CpG}$ oligodeoxynucleotide-mediated costimulation. Immunity 25: 783-793, 2006.

21. Crowell JA, Steele VE and Fay JR: Targeting the AKT protein kinase for cancer chemoprevention. Mol Cancer Ther 6 : 2139-2148, 2007.

22. Hennessy BT, Smith DL, Ram PT, Lu Y and Mills GB: Exploiting the PI3K/AKT pathway for cancer drug discovery. Nat Rev Drug Discov 4: 988-1004, 2005.

23. Paweletz CP, Charboneau L, Bichsel VE, Simone NL, Chen T, Gillespie JW, Emmert-Buck MR, Roth MJ, Petricoin III EF and Liotta LA: Reverse phase protein microarrays which capture disease progression show activation of pro-survival pathways at the cancer invasion front. Oncogene 20: 1981-1989, 2001.

24. García MG, Alaniz LD, Cordo Russo RI, Alvarez E and Hajos SE: PI3K/Akt inhibition modulates multidrug resistance and activates NF- $\kappa \mathrm{B}$ in murine lymphoma cell lines. Leuk Res 33: 288-296, 2009.

25. Filipits M: Mechanisms of cancer: multidrug resistance. Drug Discov Today Dis Mech 1: 229-234, 2004.

26. Kuo MT, Liu Z, Wei Y, Lin-Lee YC, Tatebe S, Mills GB and Unate H: Induction of human MDR1 gene expression by 2-acetylaminofluorene is mediated by effectors of the phosphoinositide 3-kinase pathway that activate NF-kB signaling. Oncogene 21: 1945-1954, 2002.

27. Barancík M, Bohácová V, Sedlák J, Sulová Z and Breier A: LY294,002, a specific inhibitor of PI3K/Akt kinase pathway, antagonizes P-glycoprotein-mediated multidrug resistance. Eur J Pharm Sci 29: 426-434, 2006.

28. Gill PK, Gescher A and Gant TW: Regulation of MDR1 promoter activity in human breast carcinoma cells by protein kinase $C$ isozymes $\alpha$ and $\theta$. Eur J Biochem 268: 4151-4157, 2001.

29. Vertegaal AC, Kuiperij HB, Yamaoka S, Courtois G, van der Eb AJ and Zantema A: Protein kinase $C-\alpha$ is an upstream activator of the IкB kinase complex in the TPA signal transduction pathway to NF- $\kappa$ B in U2OS cells. Cell Signal 12: 759-768, 2000.

30. Chao X, Zao J, Xiao-Yi G, Li-Jun M and Tao S: Blocking of $\mathrm{PI} 3 \mathrm{~K} / \mathrm{AKT}$ induces apoptosis by its effect on NF- $\mathrm{NB}$ activity in gastric carcinoma cell line SGC7901. Biomed Pharmacother 64: 600-604, 2010
31. Engelman JA: Targeting PI3K signalling in cancer: opportunities, challenges and limitations. Nat Rev Cancer 9: 550-562, 2009.

32. Nuutinen U, Postila V, Mättö M, Eeva J, Ropponen A, Eray M, Riikonen P and Pelkonen J: Inhibition of PI3-kinase-Akt pathway enhances dexamethasone-induced apoptosis in a human follicular lymphoma cell line. Exp Cell Res 312: 322-330, 2006.

33. Ghobrial IM, Witzig TE and Adjei AA: Targeting apoptosis pathways in cancer therapy. CA Cancer J Clin 55: 178-194, 2005.

34. Fulda S and Debatin KM: Extrinsic versus intrinsic apoptosis pathways in anticancer chemotherapy. Oncogene 25: 4798-4811, 2006.

35. Riedl SJ and Salvesen GS: The apoptosome: signalling platform of cell death. Nat Rev Mol Cell Biol 8: 405-413, 2007.

36. Elumalai P, Gunadharini DN, Senthilkumar K, Banudevi S, Arunkumar R, Benson CS, Sharmila G and Arunakaran J: Induction of apoptosis in human breast cancer cells by nimbolide through extrinsic and intrinsic pathway. Toxicol Lett 215: 131-142, 2012.

37. Gillings AS, Balmanno K, Wiggins CM, Johnson $\mathrm{M}$ and Cook SJ: Apoptosis and autophagy: BIM as a mediator of tumour cell death in response to oncogene-targeted therapeutics. FEBS J 276: 6050-6062, 2009.

38. Tait SW and Green DR: Mitochondria and cell death: outer membrane permeabilization and beyond. Nat Rev Mol Cell Biol 11: 621-632, 2010.

39. Kaufmann T, Strasser A and Jost PJ: Fas death receptor signalling: roles of Bid and XIAP. Cell Death Differ 19: 42-50, 2012.

40. Hussain AR, Ahmed SO, Ahmed M, Khan OS, Al Abdulmohsen S, Platanias LC, Al-Kuraya KS and Uddin S: Cross-talk between $\mathrm{NF \kappa B}$ and the PI3-kinase/AKT pathway can be targeted in primary effusion lymphoma (PEL) cell lines for efficient apoptosis. PLoS One 7: e39945, 2012.

41. Shaw RJ and Cantley LC: Ras, PI(3)K and mTOR signalling controls tumour cell growth. Nature 441: 424-430, 2006.

42. Nicholson KM and Anderson NG: The protein kinase B/Akt signalling pathway in human malignancy. Cell Signal 14: 381-395, 2002.

43. Shant J, Cheng K, Marasa BS, Wang JY and Raufman JP: Akt-dependent NF- $\kappa B$ activation is required for bile acids to rescue colon cancer cells from stress-induced apoptosis. Exp Cell Res 315: 432-450, 2009.

44. Uchida M, Iwase M, Takaoka S, Yoshiba S, Kondo G, Watanabe H, Ohashi M, Nagumo M and Shintani S: Enhanced susceptibility to tumor necrosis factor-related apoptosis-inducing ligand-mediated apoptosis in oral squamous cell carcinoma cells treated with phosphatidylinositol 3-kinase inhibitors. Int J Oncol 30: 1163-1171, 2007

45. Mantovani I, Cappellini A, Tazzari PL, Papa V, Cocco L and Martelli AM: Caspase-dependent cleavage of $170-\mathrm{kDa}$ P-glycoprotein during apoptosis of human T-lymphoblastoid CEM cells. J Cell Physiol 207: 836-844, 2006.

46. McDonald GT, Sullivan R, Paré GC and Graham CH: Inhibition of phosphatidylinositol 3-kinase promotes tumor cell resistance to chemotherapeutic agents via a mechanism involving delay in cell cycle progression. Exp Cell Res 316: 3197-3206, 2010.

47. Shingu T, Yamada K, Hara N, Moritake K, Osago H, Terashima M, Uemura T, Yamasaki T and Tsuchiya M: Growth inhibition of human malignant glioma cells induced by the PI3-K-specific inhibitor. J Neurosurg 98: 154-161, 2003. 